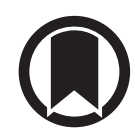

CrossMark

\title{
Similarities between COVID-19 and anti-MDA5 syndrome: what can we learn for better care?
}

\author{
To the Editor:
}

The coronavirus disease 2019 (COVID-19) pandemic has struck worldwide, leading to more than 7 million cases by June 2020 , with a $\sim 5.5 \%$ mortality rate, mainly due to acute respiratory distress syndrome (ARDS) [1].

Current conventional treatment is mainly based on support therapy and there is an urgent need for effective, specific treatments.

As yet, no direct-acting antiviral drugs have demonstrated efficacy in the disease. In contrast, increasing evidence indicates an immune-mediated pathophysiology that is paving the way for the evaluation of immunomodulation strategies [2]. In support of this view, we would like to highlight the striking similarities between COVID-19 and a rare autoimmune disease: the anti-MDA5-syndrome.

The hallmark of this disease is the presence of auto-antibodies targeting MDA5, an intracellular sensor of viral RNA (including coronavirus) that triggers the innate immune response [3]. The syndrome is characterised by systemic signs resembling COVID-19, and ARDS is the main cause of death (figure 1a) $[4,5]$. In addition, chest computed tomography findings [6], as well as blood cytokine profiles [7, 8] are very similar in the two conditions (figure $1 \mathrm{~b}$ and $\mathrm{c}$ ), further supporting common pathophysiological mechanisms. So far, there is no evidence that patients with COVID-19 have anti-MDA5 autoantibodies but, while other diseases causing ARDS feature "cytokine storm", few show such similarities with severe acute respiratory syndrome coronavirus 2 (SARS-CoV-2) infection.

Beyond these similarities, anti-MDA5 syndrome responds to glucocorticoids and immunomodulatory drugs, among which tofacitinib (a JAK inhibitor) $[9,10]$ and a combination of tacrolimus and

a)

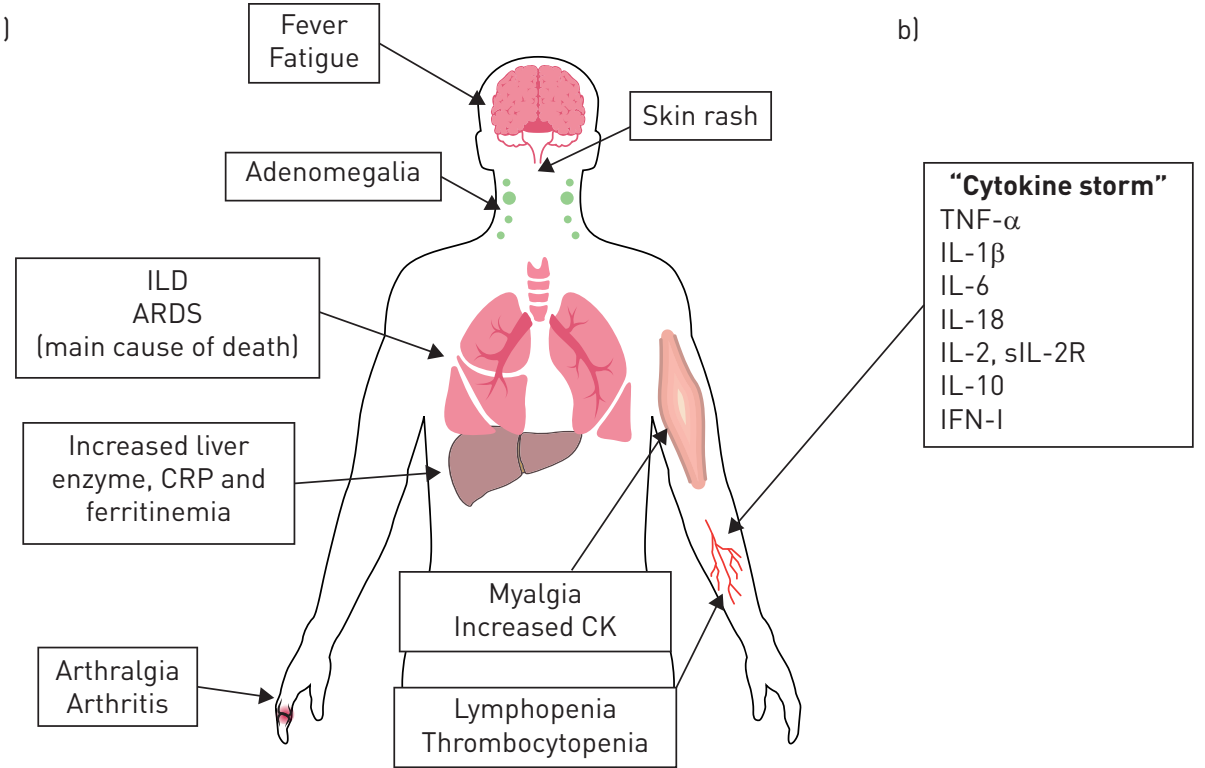

c)
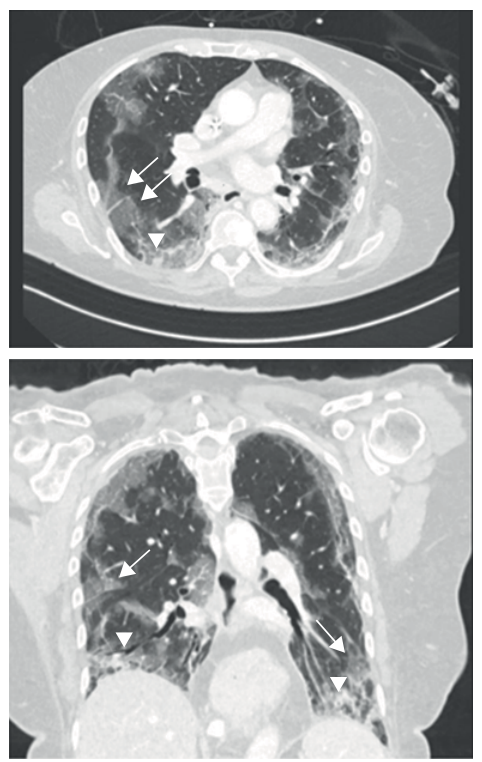

FIGURE 1 a) Clinical and biological features of anti-MDA5 syndrome. b) Cytokines whose levels are increased in anti-MDA5 syndrome patients' serum. c) High-resolution computed tomography of an anti-MDA5 syndrome patient, showing bilateral peripheral subpleural ground-glass opacities prevailing in the lower lobes (arrows), with limited consolidation (arrowheads). ILD: interstitial lung disease; ARDS: acute respiratory distress syndrome; CRP: C-reactive protein; CK: creatine kinase; TNF- $\alpha$ : tumour necrosis factor- $\alpha$; IL: interleukin; s-IL-2R: soluble IL-2 receptor; IFN-I: interferon type I. 
cyclophosphamide [11] have recently been shown to improve survival versus conventional strategies. Likewise, even though an immunological reaction is necessary to eliminate SARS-CoV-2 infection and corticosteroids are not currently recommended by the World Health Organization [12], in severe COVID-19 patients, dexamethasone has been reported to improve survival [13] and ruxolitinib (another JAK inhibitor) results in a greater chest tomography improvement and faster clinical improvement with no death compared with standard of care [14]. Moreover, tacrolimus has been shown to inhibit SARS-CoV replication [15].

These data support further evaluation of employing such an immunomodulatory strategy in COVID-19.

@ERSpublications

Similarities between COVID-19 and anti-MDA5 syndrome support further evaluation of employing an immunomodulatory strategy in COVID-19 https://bit.ly/3dN6lJ8

Cite this article as: Giannini M, Ohana M, Nespola B, et al. Similarities between COVID-19 and anti-MDA5 syndrome: what can we learn for better care?. Eur Respir J 2020; 56: 2001618 [https://doi.org/ 10.1183/13993003.01618-2020].

Margherita Giannini ${ }^{1}$, Mickael Ohana ${ }^{2}$, Benoit Nespola $^{3}$, Giovanni Zanframundo ${ }^{4,5}$, Bernard Geny ${ }^{1,6}$ and Alain Meyer ${ }^{1,6,7}$

${ }^{1}$ Physiology and Functional Explorations Dept, University Hospitals of Strasbourg, Strasbourg, France. ${ }^{2}$ Radiology Dept, Nouvel Hôpital Civil, University Hospitals of Strasbourg, Strasbourg, France. ${ }^{3}$ Laboratory of Immunology, Nouvel Hôpital Civil, University Hospitals of Strasbourg, Strasbourg, France. ${ }^{4}$ Rheumatology, Fondazione IRCCS Policlinico San Matteo, Pavia, Italy. ${ }^{5}$ Dept of Internal Medicine and Medical Therapeutics, University of Pavia, Pavia, Italy. ${ }^{6}$ Federation of Translational Medicine of Strasbourg, Strasbourg University, Strasbourg, France. ${ }^{7}$ Rheumatology Dept, National Centre for Rare Systemic Autoimmune Diseases, University Hospitals of Strasbourg, Strasbourg, France.

Correspondence: Alain Meyer, Rheumatology Dept, National Centre for Rare Systemic Autoimmune Diseases, University Hospitals of Strasbourg, 1 avenue Molière, Strasbourg 67098, France. E-mail: alain.meyer1@chru-strasbourg.fr

Received: 6 May 2020 | Accepted after revision: 25 June 2020

Acknowledgements: We thank Pragnell Babette for her English support.

Author contributions: M. Giannini: literature search, figures, study design, data collection, data analysis, data interpretation, writing. M. Ohana: figures, data collection, data interpretation. B. Nespola: data interpretation. G. Zanframundo: data interpretation. B. Geny: data interpretation, writing. A. Meyer: literature search, figures, study design, data collection, data analysis, data interpretation, writing.

Conflict of interest: None declared.

References

1 WHO. Coronavirus disease (COVID-19) situation report - 106. www.who.int/emergencies/diseases/novelcoronavirus-2019/situation-reports/ Date last updated: 5 May 2020. Date last accessed: 5 May 2020.

2 Mehta P, McAuley DF, Brown M, et al. COVID-19: consider cytokine storm syndromes and immunosuppression. The Lancet 2020; 395: 1033-1034.

3 Dias Junior AG, Sampaio NG, Rehwinkel J. A balancing act: MDA5 in antiviral immunity and autoinflammation. Trends Microbiol 2019; 27: 75-85.

4 Allenbach Y, Uzunhan Y, Toquet S, et al. Different phenotypes in dermatomyositis associated with anti-MDA5 antibody: study of 121 cases. Neurology 2020; 95: e70-e78.

5 Li J, Liu Y, Li Y, et al. Associations between anti-melanoma differentiation-associated gene 5 antibody and demographics, clinical characteristics and laboratory results of patients with dermatomyositis: a systematic meta-analysis. J Dermatol 2018; 45: 46-52.

6 Tanizawa K, Handa T, Nakashima R, et al. HRCT features of interstitial lung disease in dermatomyositis with anti-CADM-140 antibody. Respir Med 2011; 105: 1380-1387.

7 Gono T, Kaneko H, Kawaguchi Y, et al. Cytokine profiles in polymyositis and dermatomyositis complicated by rapidly progressive or chronic interstitial lung disease. Rheumatology 2014; 53: 2196-2203.

8 Nishioka A, Tsunoda S, Abe T, et al. Serum neopterin as well as ferritin, soluble interleukin-2 receptor, KL-6 and anti-MDA5 antibody titer provide markers of the response to therapy in patients with interstitial lung disease complicating anti-MDA5 antibody-positive dermatomyositis. Mod Rheumatol 2019; 29: 814-820.

9 Chen Z, Wang X, Ye S. Tofacitinib in amyopathic dermatomyositis-associated interstitial lung disease. $N$ Engl J Med 2019; 381: 291-293.

10 Kurasawa K, Arai S, Namiki Y, et al. Tofacitinib for refractory interstitial lung diseases in anti-melanoma differentiation-associated 5 gene antibody-positive dermatomyositis. Rheumatology 2018; 57: 2114-2119.

11 Tsuji H, Nakashima R, Hosono Y, et al. Multicenter prospective study of the efficacy and safety of combined immunosuppressive therapy with high-dose glucocorticoid, tacrolimus, and cyclophosphamide in interstitial lung diseases accompanied by anti-melanoma differentiation-associated gene 5-positive dermatomyositis. Arthritis Rheumatol 2020; 72: 488-498.

12 WHO. Clinical management of COVID-19: interim guidance. www.who.int/publications/i/item/clinicalmanagement-of-covid-19 Date last updated: 27 May 2020. Date last accessed: 27 May 2020. 
13 Ledford H. Coronavirus breakthrough: dexamethasone is first drug shown to save lives. Nature 2020; $582: 469$.

14 Cao Y, Wei J, Zou L, et al. Ruxolitinib in treatment of severe coronavirus disease 2019 (COVID-19): a multicenter, single-blind, randomized controlled trial. J Allergy Clin Immunol 2020; 146: 137-146.

15 Carbajo-Lozoya J, Müller MA, Kallies S, et al. Replication of human coronaviruses SARS-CoV, HCoV-NL63 and HCoV-229E is inhibited by the drug FK506. Virus Res 2012; 165: 112-117.

Copyright (CERS 2020 . This version is distributed under the terms of the Creative Commons Attribution Non-Commercial Licence 4.0. 